\title{
Vitamina D nativa e/o attiva in dialisi
}

\author{
Andrea Galassi
}

U.O. Nefrologia e Dialisi, A.O. Desio-Vimercate, Desio (MB)

\begin{abstract}
ACtive ANd/OR NATIVE VITAMin D FOR DiALYSIS PATIENTS
Abstract. Active vitamin D represents a relevant treatment for CKD-MBD. Recent data suggest new applications of active vitamin $D$ beyond secondary hyperparathyroidism, such as the control of albuminuria and left ventricular hypertrophy in diabetic patients with CKD stage 3-4. Native vitamin D has contemporarily received a growing interest due to several pleiotropic effects, potentially induced at autocrine-paracrine level, even in the late stages of CKD. However, the efficacy of vitamin $D$ in terms of clinically relevant outcome is still based on observational data. The present review summarizes the evidences and the unanswered questions concerning the use of vitamin D in CKD-MBD, with special attention to the rationale for native vitamin $D$ administration in dialysis patients.
\end{abstract}

Key words: Native vitamin D, VDRA, Cholecalciferol, Ergocalciferol, Calcifediol, Dialysis, CKD-MBD

Conflict of interest: Andrea Galassi has received occasional speaker's fees from AbbVie and Shire.

Financial support: The author has received no financial support for the preparation of this manuscript.

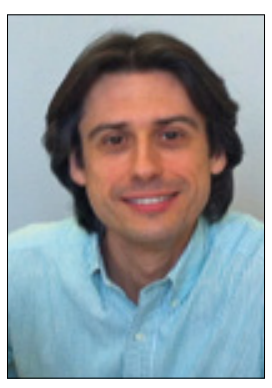

\section{Introduzione}

La Vitamina D costituisce un caposaldo delle terapie contro l'iperparatiroidismo secondario (IPS) in corso di insufficienza renale cronica (IRC). La recente storia della Nefrologia ha conosciuto le complicanze severe dell'IPS, come i tumori bruni, la cardiopatia ipertrofica, i dolori ossei, le fratture e la calcifilassi. Benché l'esperienza clinica ci porti quotidianaAndrea Galassi mente al trattamento e alla prevenzione dell'IPS, a oggi non sono disponibili studi randomizzati controllati ( $\mathrm{RCT}$ ) che dimostrino se una riduzione farmacologica dei livelli di PTH offra reali vantaggi in termini di ospedalizzazioni, eventi cardiovascolari, progressione dell'IRC e sopravvivenza. Infatti, l'associazione indipendente tra elevati livelli di PTH ed eventi infausti è limitata a studi osservazionali (1). Le Linee Guida KDIGO suggeriscono di mantenere i livelli di $\mathrm{PTH}$ all'interno dei range di normalità nell'IRC agli stadi 3-5 e tra 2-9 volte i valori normali nell'end stage renal disease (ESRD) (2). Tuttavia, l'incertezza riguardo ai livelli ottimali di PTH in corso di IRC limita considerevolmente il grado di evidenza che sostiene tale suggerimento (2). Gli attivatori della Vitamina D (Vitamin D Receptor Activator, VDRA) sono una delle terapie consigliate per il raggiungimento dei target di PTH (2). Allo stesso tempo, le Linee Guida KDIGO suggeriscono di correggere livelli di $25(\mathrm{OH})$
D inferiori a $30 \mathrm{ng} / \mathrm{mL}$ con ergocalciferolo come primo step nel trattamento dell'IPS nell'IRC agli stadi 3-5 (2). Tuttavia, questo suggerimento non è stato corredato da alcun grado di evidenza e non si estende ai pazienti dializzati (2).

La scelta tra l'utilizzo della Vitamina D attiva e/o nativa è oggi sempre più complessa. Da un lato, la rapida nascita di alcuni VDRA, ciascuno dotato di azioni peculiari, non è stata accompagnata da studi di confronto testa a testa che abbiano fornito sicure conclusioni riguardo al loro differente impatto sulla CKD-MBD. Allo stesso tempo, la Vitamina D nativa è stata al centro di un crescente interesse per i suoi potenziali effetti pleiotropici autocrino-paracrini anche in corso di IRC. L'articolo sintetizza le evidenze e le domande ancora aperte sul trattamento con Vitamina D nella CKD-MBD, con particolare attenzione all'attuale razionale per l'impiego della Vitamina $\mathrm{D}$ nativa in dialisi.

\section{La Vitamina D nativa}

Con il termine "Vitamina D nativa" si fa riferimento alla $25(\mathrm{OH}) \mathrm{D}$, naturale precursore della Vitamina $\mathrm{D}$ attiva $\left(1-25(\mathrm{OH})_{2} \mathrm{D}\right.$ o calcitriolo). La $25(\mathrm{OH}) \mathrm{D}$ deriva, a sua volta, dall'idrossilazione epatica in posizione 25 dei suoi due precursori: l'ergocalciferolo, con la formazione della $25(\mathrm{OH}) \mathrm{D}_{2}$, e il colecalciferolo, con la formazione della $25(\mathrm{OH}) \mathrm{D}_{3}$. L'ergocalciferolo ha origine dall'irradiazione UV dell'ergosterolo presente nei lieviti, naturalmente contenuto nei funghi, mentre il colecalciferolo dall'irradiazione del 7-deidrocolesterolo (3). 


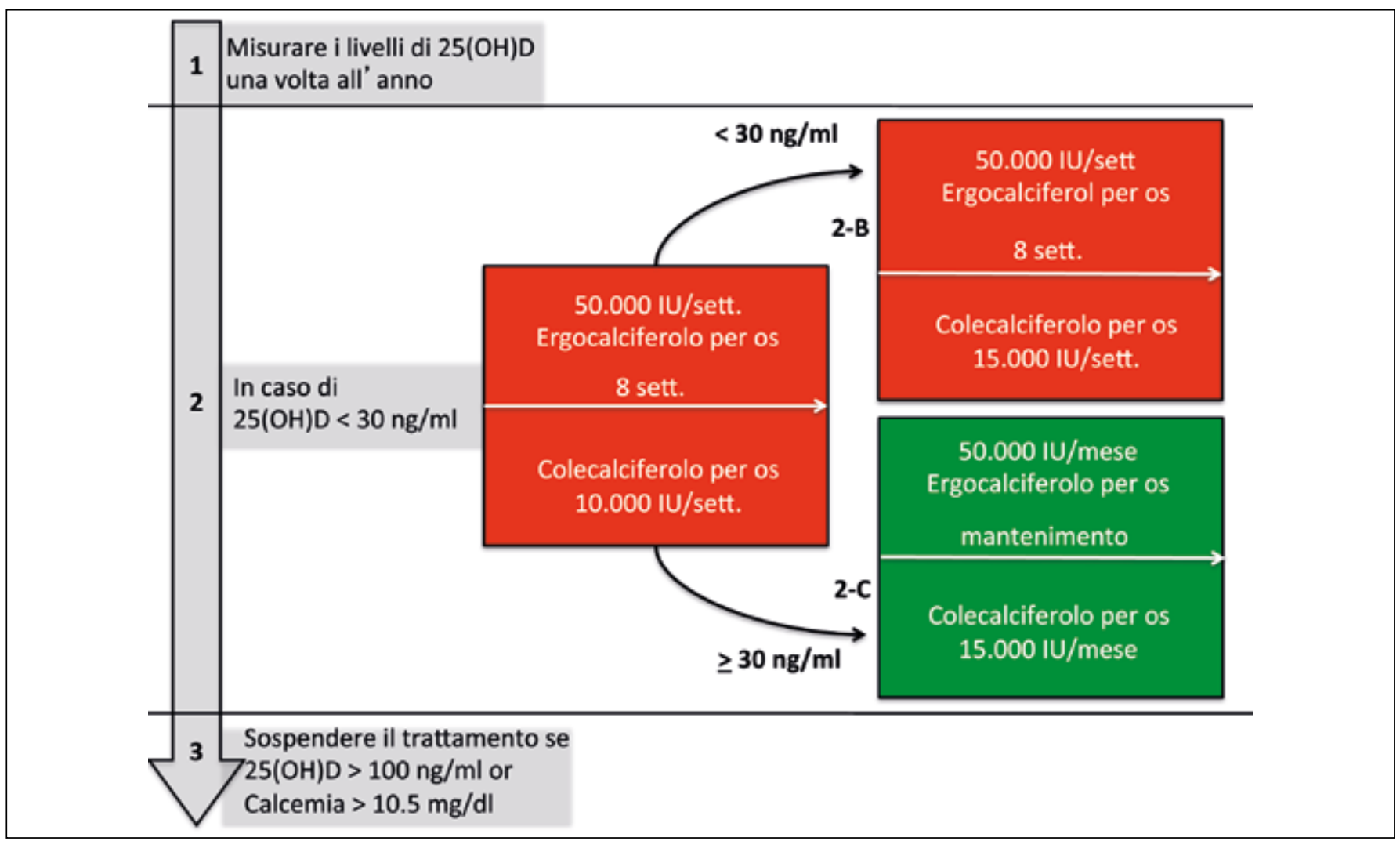

Fig. 1 - Possibile schema di correzione del deficit di 25(OH)D secondo Nigwekar et al. (22).

1) Misurare i livelli di $25(\mathrm{OH}) \mathrm{D}$ una volta all'anno; 2 ) in caso di livelli di $25(\mathrm{OH}) \mathrm{D}$ inferiori a $30 \mathrm{ng} / \mathrm{mL}$ procedere con un ciclo di 2 mesi con ergocalciferolo orale (50.000 UI/sett) o con colecalciferolo orale (10.000 UI/sett), quindi ricontrollare i livelli di $25(\mathrm{OH}) \mathrm{D}$; $2 \mathrm{~B}) \mathrm{se}$ i livelli sono ancora <30 ng/mL procedere con un secondo ciclo di 2 mesi con ergocalciferolo (50.000 UI/sett) o con colecalciferolo a dosaggio superiore (15.000 UI/sett); al termine delle 8 settimane non si consiglia di ripetere il dosaggio della $25(\mathrm{OH}) \mathrm{D}$ perché il secondo ciclo dovrebbe essere sufficiente per correggere il deficit; $2 \mathrm{C}$ ) una volta raggiunti livelli di $25(\mathrm{OH}) \mathrm{D} \geq 30 \mathrm{ng} / \mathrm{mL}$ impostare il mantenimento con ergocalciferolo (50.000 $\mathrm{UI} / \mathrm{mese})$ o con colecalciferolo (15.000 UI/mese o 1.000-2.000 UI/die); 3) sospendere il trattamento con Vitamina D nativa in caso di livelli di $25(\mathrm{OH}) \mathrm{D}>100 \mathrm{ng} / \mathrm{mL} \mathrm{o} \mathrm{calcemia}>10.5 \mathrm{mg} / \mathrm{dL}$.

Si ricordi che l'uomo non può sintetizzare la $25(\mathrm{OH}) \mathrm{D}_{2}(3) \mathrm{e}$ che 1'80\% della Vitamina D umana deriva dall'irradiazione solare con una minima quota di origine alimentare. $\mathrm{La} 25(\mathrm{OH})$ $\mathrm{D}$ è, infine, attivata a $1-25(\mathrm{OH})_{2} \mathrm{D}$ dalle 1 -alfa-idrossilasi renali ed extra-renali (4).

La carenza di $25(\mathrm{OH}) \mathrm{D}$ (livelli circolanti $<30 \mathrm{ng} / \mathrm{mL}$ ) viene più precisamente classificata in "insufficienza" $(25(\mathrm{OH}) \mathrm{D}$ tra 21 e $29 \mathrm{ng} / \mathrm{mL})$ e in "deficit" $(25(\mathrm{OH}) \mathrm{D}<20 \mathrm{ng} / \mathrm{mL})(4)$. Ripetute analisi osservazionali hanno descritto associazioni tra bassi livelli circolanti di $25(\mathrm{OH}) \mathrm{D}$ e svariate condizioni patologiche nella popolazione generale, come l'osteoporosi (5), l'ipertensione (6), gli eventi cardiovascolari (7), il diabete (8), le infezioni (9), il cancro (10) e la mortalità (11). Così, anche nei pazienti con IRC, i livelli di $25(\mathrm{OH}) \mathrm{D}$ sono risultati associati alla progressione della malattia renale (12), all'IPS (13) e a una ridotta sopravvivenza (12). Questi dati hanno suggerito che la $25(\mathrm{OH}) \mathrm{D}$ possa costituire un effettore protettivo contro eventi clinici sfavorevoli e non solo un marcatore di malattia. Il razionale per un simile effetto benefico è sostenuto dai potenziali effetti pleiotropici a livello autocrino e paracrino, che potrebbero esprimersi anche nell'ESRD grazie all'attività delle 1-alfa-idrossilasi extra-renali (14). Tuttavia, queste le- gittime aspettative richiedono una cauta valutazione prima di condurre a solidi orientamenti terapeutici.

Le Linee Guida KDIGO suggeriscono la correzione dei livelli di $25(\mathrm{OH}) \mathrm{D}<30 \mathrm{ng} / \mathrm{mL}$ nell'IRC agli stadi 3-5 (2). Tuttavia, i target ottimali di $25(\mathrm{OH}) \mathrm{D}$ nell'IRC (5) e le strategie per correggere il deficit di Vitamina D restano incerti. Sono attualmente disponibili tre forme di Vitamina D nativa: il calcifediolo e i suoi due precursori, l'ergocalciferolo e il colecalciferolo, che necessitano anche dell'idrossilazione epatica in posizione 25. In alcuni lavori, l'ergocalciferolo è risultato meno potente del colecalciferolo nel raggiungimento dei target di $25(\mathrm{OH})$ D (15). Questa differenza sembra dovuta alla maggiore affinità del colecalciferolo per la vitamin D binding protein e alla prolungata attivazione del recettore della Vitamina D (Vitamin D Receptor, VDR) da parte della $1-24-25(\mathrm{OH}) \mathrm{D}_{3}$ rispetto alla $1-24-25(\mathrm{OH}) \mathrm{D}_{2}(15)$. Si ritiene, quindi, che, in termini dose-risposta, 50.000 UI di ergocalciferolo corrispondano a 15.000-5.000 UI di colecalciferolo (16). Due RCT stanno attualmente confrontando l'effetto del colecalciferolo e dell'ergocalciferolo sul metabolismo minerale nell'IRC agli stadi 2-5 (NCT01633853, NCT01173848). Va, inoltre, ricordato che il rischio di ipercalcemia potrebbe essere inferiore con $\mathrm{i}$ 


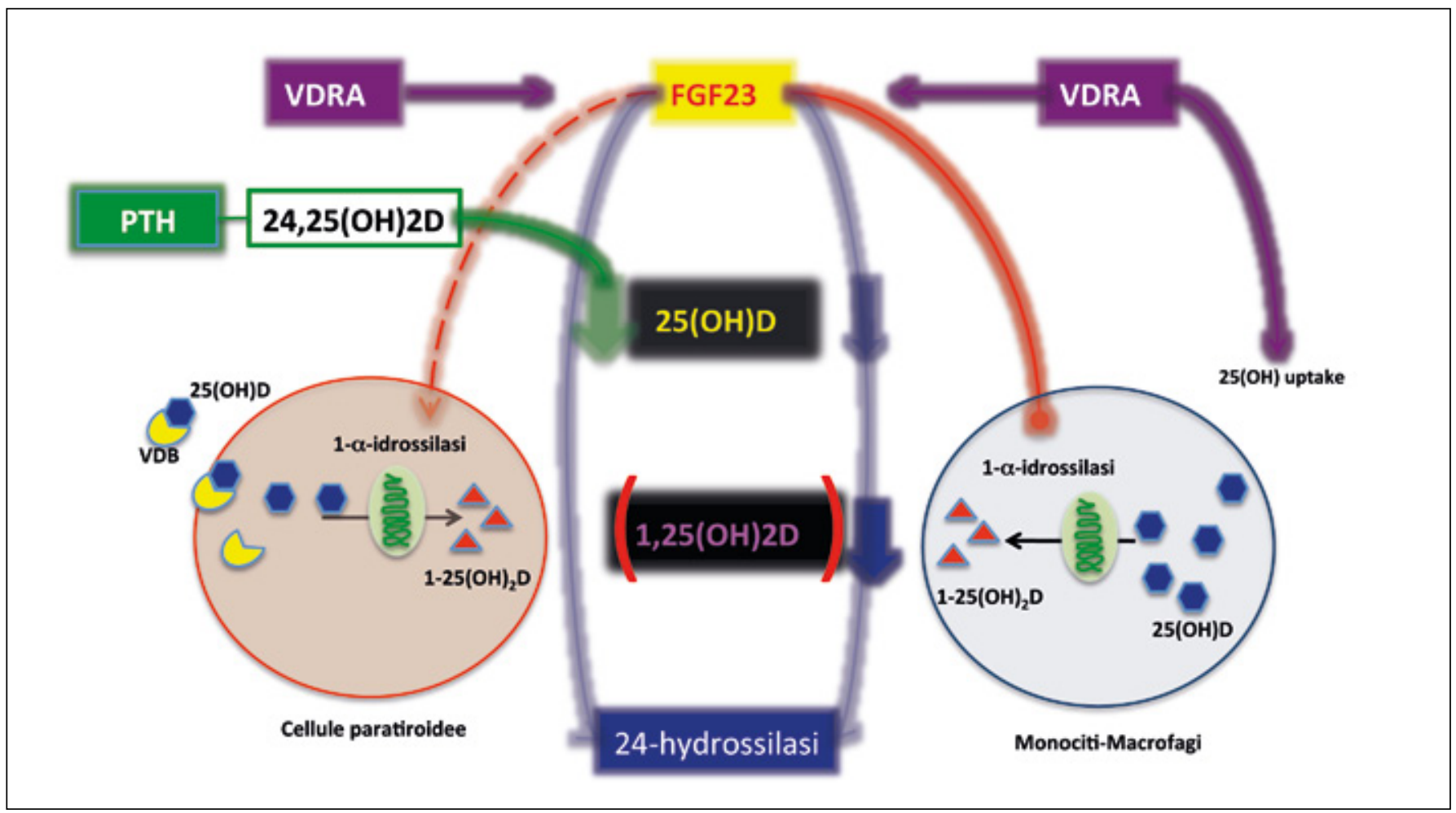

Fig. 2 - Metabolismo e azioni della 25(OH)D in corso di IRC.

Alti livelli di PTH, di FGF23 e la concomitante somministrazione di Vitamina D attiva (Vitamin D Receptor Activator, VDRA) possono alterare il metabolismo della 25(OH)D in corso di IRC. Elevate concentrazioni di PTH e di FGF23 possono stimolare il catabolismo della 25(OH)D tramite l'attivazione della 24-idrossilasi. FGF23 ha, inoltre, un'azione opposta sulla l'1- $\alpha$-idrossilasi dei monociti-macrofagi (inibizione) e delle cellule paratiroidee (induzione). I VDRA possono, a loro volta, inibire le 1 - $\alpha$-idrossilasi e aumentare direttamente i livelli di FGF23.

Ulteriori ricerche dovranno studiare l'interazione tra queste vie metaboliche e l'efficacia terapeutica della Vitamina D nativa, sola o in associazione con VDRA, nei pazienti uremici.

due pro-farmaci, ergocalciferolo e colecalciferolo, rispetto al calcifediolo. Cautela va sempre posta nel sospetto di condizioni predisponenti all'iperattività delle 1-alfa-idrossilasi extrarenali, come la sarcoidosi e i linfomi a cellule $\mathrm{B}$. L'attività della 25-idrossilasi epatica sembra, invece, conservata nei pazienti cirrotici, dove la somministrazione di colecalciferolo può correggere efficacemente il deficit di $25(\mathrm{OH}) \mathrm{D}(17)$. Thadhani et al. hanno suggerito di estendere l'indicazione alla correzione del deficit di 25(OH)D anche nell'ESRD, partendo con 50.000 UI di ergocalciferolo o 10.000 UI di colecalciferolo alla settimana per due mesi, seguiti da una terapia di mantenimento (Fig. 1) (16).

La recente metanalisi di Kandula et al. ha descritto una riduzione nei livelli di PTH di circa $60 \mathrm{pg} / \mathrm{mL}$ in 746 dializzati trattati con Vitamina D nativa (18). La variazione del PTH è risultata comunque inferiore rispetto a quanto osservato con Vitamina D attiva nella metanalisi Cochrane $(-196.05 \mathrm{pg} / \mathrm{mL})$ (19). L'entità della soppressione del PTH descritta con la Vitamina D nativa suggerisce come la correzione del suo deficit potrebbe essere preventiva o terapeutica nelle forme lievi o iniziali di IPS (20). Tuttavia, il confronto tra Vitamina D nativa e VDRA nel controllo dell'IPS richiede ulteriori approfondimenti in futuri RCT.
L'espressione ubiquitaria delle 1-alfa-idrossilasi extra-renali ha alimentato crescenti speranze riguardo ai potenziali effetti pleiotropici della Vitamina D nativa anche nei pazienti dializzati (14). Va, però, considerato che l'attivazione extra-renale della $25(\mathrm{OH}) \mathrm{D}$ potrebbe essere down-regolata dagli elevati livelli di PTH e di FGF23 tipici dell'ESRD nonché dalla concomitante terapia con VDRA (Fig. 2). Di fatto, recenti studi hanno segnalato un effetto benefico del colecalciferolo sull'ipertrofia ventricolare sinistra (IVS) (21), sulle dosi di eritropoietina (22) e sull'infiammazione (23) e alcuni RCT stanno investigando sull'impatto della terapia con Vitamina D nativa sulle dosi di EPO (NCT01395823), sul sistema immunitario (NCT00892099), sull'insulino resistenza (NCT00893451) e sulla performance cognitiva (NCT00511225, NCT01229878) nell'IRC. Tuttavia, allo stato dell'arte, le evidenze scientifiche non hanno ancora dimostrato se la terapia con Vitamina D nativa possa realmente esprimere i suoi effetti pleiotropici anche in ESRD.

Grande interesse è anche rivolto all'associazione tra livelli di 25(OH)D e sopravvivenza. La recente metanalisi di Pilz et al. ha descritto, nei pazienti nefropatici, un aumento del rischio di mortalità pari al $14 \%$ per ogni $10 \mathrm{ng} / \mathrm{mL}$ di riduzione nei livelli circolanti di 25(OH)D (23). Tuttavia, a oggi, nessun RCT ha 
dimostrato se la somministrazione di Vitamina D nativa possa favorire una migliore sopravvivenza nell'IRC. Peraltro, esistono alcuni dubbi riguardo al significato dell'associazione tra livelli di Vitamina D nativa e mortalità nei pazienti dializzati in trattamento con VDRA (24). Possibili risposte potrebbero giungere dallo studio italiano NUTRIVITA, attualmente in fase di randomizzazione, disegnato per testare l'effetto del calcifediolo versus placebo sulla sopravvivenza, sull'infarto miocardico non fatale e sull'ictus in dialisi (NCT01457001). Nell'attesa di nuovi RCT, il suggerimento KDIGO verso la correzione del deficit di $25(\mathrm{OH}) \mathrm{D}$ sembra ragionevole come terapia adiuvante in corso di IPS, specialmente nelle sue fasi iniziali. Non sembrano, peraltro, sussistere controindicazioni alla somministrazione di Vitamina D nativa in dialisi. Tuttavia, molte domande restano in attesa di risposte riguardo ai livelli ottimali di $25(\mathrm{OH}) \mathrm{D}$ in corso di IRC e all'impatto clinico che la terapia con Vitamina $\mathrm{D}$ nativa potrà offrire su rilevanti outcome clinici come l'incidenza di eventi cardiovascolari, fratture, diabete, infezioni, ospedalizzazioni e mortalità nell'ESRD.

\section{La Vitamina D attiva}

I VDRA sono comunemente utilizzati nel trattamento dell'IPS nell'IRC. Tuttavia, il grado di evidenza che ne supporta l'impiego è ancora scarso, data l'assenza di RCT che ne abbiano dimostrato la superiorità rispetto al placebo su patient-centered outcome oltre che sul controllo dei parametri bioumorali (PTH, calcemia e fosforemia).

Le alte dosi di VDRA, talora necessarie nei casi di grave IPS, possono indurre ipercalcemia e iperfosforemia con ricadute negative sulle calcificazioni dei tessuti molli. L'industria farmaceutica ha, quindi, sviluppato alcuni VDRA selettivi capaci di inibire i livelli di PTH con un impatto sul sovraccarico di calcio $(\mathrm{Ca})$ e fosforo $(\mathrm{P})$ inferiore rispetto al calcitriolo $(25) \mathrm{e}$, in alcune casistiche, sovrapponibile al placebo (26). Sono oggi disponibili diversi analoghi sintetici sia della Vitamina D2 (paracalcitolo e doxercalciferolo) sia della Vitamina D3 (alfacalcidiolo, falecalcitriolo e maxacalcitolo). Dati sperimentali suggeriscono come ogni analogo agisca in modo peculiare sui recettori della Vitamina D. Alcuni modelli animali, per esempio, hanno dimostrato un minore impatto sulle calcificazioni vascolari (27) e sul basso turnover osseo con il paracalcitolo rispetto ad altri VDRA (28). Tuttavia, simili effetti sono ancora inesplorati nell'uomo. Inoltre, il confronto diretto tra i vari VDRA in termini di controllo di PTH, Ca e P è ancora limitato a pochi studi d'intervento con risultati eterogenei (29-31). Così come la Vitamina D nativa, anche i VDRA sono stati al centro di un crescente interesse riguardo agli effetti pleiotropici legati alla regolazione genica offerta dall'attivazione ubiquitaria dei VDR $(32,33)$. Gli studi VITAL (34) e PRIMO (35) hanno recentemente dimostrato un effetto incoraggiante del paracalcitolo rispetto al placebo in aggiunta ad ACE-I e ARB nel controllo dell'albuminuria e dell'IVS nei pazienti diabetici con IRC agli stadi 3-4. Tuttavia, le conoscenze cliniche riguardo agli effetti pleiotropici dei VDRA in dialisi sono ancora preliminari. Lo studio PRIMO 2 (NCT00616902), disegnato per testare l'effetto del paracalcitolo rispetto al pla- cebo sull'IVS nell'ESRD, è stato recentemente interrotto per difficoltà di arruolamento.

Alcuni studi osservazionali hanno fornito dati eterogenei riguardo all'associazione tra la terapia con VDRA e un minor rischio di ospedalizzazioni e di morte rispetto al calcitriolo. Il paracalcitolo è risultato associato a un rischio di ospedalizzazione inferiore del 14\% rispetto al calcitriolo (36), così come altre casistiche hanno riportato una migliore sopravvivenza nei pazienti trattati con paracalcitolo (37) o doxercalciferolo (37) rispetto al calcitriolo. Tuttavia, tali risultati non sono stati poi confermati nell'analisi dello studio DOPPS, più affidabile in termini di fattori confondenti non misurabili (38). Più recentemente, lo studio FARO ha osservato una migliore sopravvivenza nei pazienti dializzati in terapia con VDRA anche in presenza di livelli di PTH $<150 \mathrm{pg} / \mathrm{mL}$ (39). La mancanza di RCT limita comunque ogni conclusione riguardo all'effetto dei VDRA sulla sopravvivenza nell'ESRD.

I VDRA restano, quindi, una delle principali terapie consigliate nel trattamento dell' IPS in dialisi. Tuttavia, siamo ancora lontani da una visione olistica riguardo alle peculiarità che contraddistinguono i VDRA oggi disponibili e al loro impatto sui più importanti outcome clinici.

\section{Vitamina D nativa \& attiva}

Il razionale per una terapia di associazione con Vitamina D nativa e attiva in dialisi è ancora incerto. Allo stato dell'arte, nessuno studio RCT ha debitamente valutato gli effetti della somministrazione simultanea di entrambe le forme di Vitamina D in termini di controllo della CKD-MBD e di effetti pleiotropici. Le nostre conoscenze sono, per ora, limitate ad analisi secondarie di pochi studi osservazionali senza gruppi di controllo (40) e perlopiù disegnati per valutare l'effetto della Vitamina D nativa sui parametri del metabolismo minerale, nei quali una quota di partecipanti era già in trattamento con VDRA (40). Il calcifediolo è risultato associato a una significativa riduzione dei valori di PTH e a un calo progressivo nella prescrizione di alfacalcidiolo (dal 66\% al 43\%) in 149 pazienti emodializzati durante 6 mesi di follow up (41). Al contrario, il colecalciferolo alla dose di $20.000 \mathrm{UI} /$ settimana non ha sortito una variazione significativa nei livelli di PTH in 64 emodializzati, 40 dei quali erano già in trattamento con alfacalcidiolo o calcitriolo (42). L'aggiunta di colecalciferolo (10.333 UI/sett) in 42 dializzati trattati con VDRA non ha prodotto una significativa riduzione dei livelli di PTH (43). Lo studio di Matias et al. ha valutato un anno di terapia con il colecalciferolo a dosi crescenti (un massimo di $50.000 \mathrm{UI} /$ sett) e calibrate sui valori basali di $25(\mathrm{OH}) \mathrm{D}$ in 158 dializzati, di cui il $44 \%$ in terapia con paracalcitolo (44). È stata osservata una significativa riduzione dei livelli di PTH (da 233 a 208 pg/mL, p<0.001), che, anche se clinicamente poco rilevante, si accompagnava a una lieve riduzione delle dosi di paracalcitolo (44). Un breve ciclo di colecalciferolo ad altissime dosi $(200.000 \mathrm{UI} /$ sett per 3 settimane) è risultato simile al placebo in termini di riduzione del PTH a 6 settimane, nonostante l'incremento medio nei valori di $25(\mathrm{OH}) \mathrm{D}$ sino a $52.4 \pm 18 \mathrm{ng} / \mathrm{mL}(45)$. Infine, un anno di trattamento con colecalciferolo alla dose di 25.000 UI ogni 15 giorni ha prodotto una significativa riduzione del PTH rispetto 
al placebo in 43 emodializzati [-115 pg/mL (95\% CI: -192-81) versus placebo $+80 \mathrm{pg} / \mathrm{mL}$ (95\% CI:-58-153), $\mathrm{p}=0.02]$ (46). Si noti, però, come alcuni lavori abbiano osservato una significativa tendenza all'ipercalcemia in corso di trattamento con Vitamina D nativa $(41,42)$.

Le note incertezze riguardo all'impatto clinico dei potenziali effetti pleiotropici della Vitamina D nativa nell'ESRD sono ulteriormente complicate nei pazienti in trattamento con VDRA. Da un lato, la tendenza verso una riduzione del PTH in corso di Vitamina D nativa negli studi appena citati suggerisce come la conversione della forma nativa in $1-25(\mathrm{OH})_{2} \mathrm{D}$ potrebbe essere conservata anche nei pazienti in terapia con VDRA. D'altra parte, nello stesso lavoro di Matias et al. i livelli di $1-25(\mathrm{OH})_{2} \mathrm{D}$ aumentavano solo nei pazienti non trattati con paracalcitolo $(\mathrm{p}<0.001)(44)$. I VDRA potrebbero, infatti, inibire l'espressione delle 1-alfa-idrossilasi extra-renali direttamente o attraverso l'aumento di FGF23 (Fig. 2) $(47,48)$. Allo stesso tempo, va ricordato come i livelli circolanti di $1-25(\mathrm{OH})_{2} \mathrm{D}$ siano poco rappresentativi delle concentrazioni intracellulari ed extravascolari, coinvolte nei processi autocrino-paracrini su cui si basa il razionale del pleiotropismo ubiquitario.

In conclusione, alcuni dati suggeriscono un potenziale effetto adiuvante della terapia con Vitamina D nativa in associazione ai VDRA per la correzione dell'IPS nell'ESRD. Tuttavia, i risultati sono ancora molto eterogenei e richiedono prossime verifiche in adeguati RCT. L'azione pleiotropica della Vitamina D nativa nell'ESRD nei pazienti in trattamento con VDRA è, al contrario, un campo quasi interamente inesplorato in attesa di risposte in ambito clinico e sperimentale.

\section{Conclusioni}

Nonostante il suo diffuso impiego in Nefrologia, la Vitamina $\mathrm{D}$ resta ancora un mondo paradossalmente poco conosciuto. Dati sperimentali ed evidenze cliniche preliminari suggeriscono un rapido allargamento dei fronti terapeutici che la Vitamina $\mathrm{D}$ tenterà presto di varcare anche nell'IRC. Tuttavia, le evidenze scientifiche non possono ancora sostenere forti raccomandazioni riguardo alle strategie terapeutiche con Vitamina D, né in termini di tipo né in termini di dosaggio, in corso di insufficienza renale. La correzione del deficit di 25(OH)D nell'IRC è ancora fondata sull'opinione, nonostante alcuni dati preliminari ne suggeriscano l'azione adiuvante nel controllo dell'IPS. La "migliore" ricetta con Vitamina D in dialisi resta, quindi, oggetto di discussione nell'attesa di nuovi RCT.

\section{Riassunto}

La terapia con Vitamina D attiva rappresenta un capitolo importante nel trattamento della CKD-MBD e recenti evidenze ne suggeriscono l'impiego anche in ambiti che valicano il semplice iperparatiroidismo secondario, come il controllo della proteinuria e dell'ipertrofia ventricolare sinistra nei pazienti diabetici con insufficienza renale cronica (IRC) agli stadi 3 e 4 . Allo stesso tempo, la Vitamina D nativa è stata al centro di un crescente interesse per i suoi effetti pleiotropici potenzialmente espressi a livello autocrino e paracrino anche nelle fasi più avanzate dell'IRC. Tuttavia, l'efficacia della Vitamina D su outcome clinici di rilievo si fonda ancora su dati prevalentemente osservazionali. Questa review sintetizza le evidenze e le domande ancora aperte sulla terapia con Vitamina D nella CKD-MBD, con particolare attenzione al razionale verso l'impiego della Vitamina D nativa in dialisi.

Parole chiave: Vitamina D nativa, VDRA, Colecalciferolo, Ergocalciferolo, Calcifediolo, Dialisi, CKD-MBD

Contributi economici agli Autori: L'Autore non ha ricevuto alcun compenso economico per la stesura del presente manoscritto.

Dichiarazione di conflitto di interessi: Andrea Galassi ha ricevuto compensi occasionali da Abbvie e Shire in qualità di speaker.

\author{
Indirizzo degli Autori: \\ Dr. Andrea Galassi \\ U.O. Nefrologia e Dialisi \\ A.O. Desio-Vimercate \\ Via Mazzini 1 \\ 20033 Desio (MB) \\ andrea-galassi@libero.it
}

\section{Bibliografia}

1. 1 Block GA, Klassen PS, Lazarus M et al. Mineral metabolism, mortality, and morbidity in maintenance hemodialysis. $\mathrm{J}$ Am Soc Nephrol 2004; 15: 2208-18.

2. KDIGO. Treatment of CKD-MBD targeted at lowering high serum phosphorus and maintaining serum calcium. Kidney Int 2009; 76 (Suppl. 113): S70-89.
3. Nair R, Maseeh A. Vitamin D: the "sunshine" vitamin. J Pharmacol Pharmacother 2012; 3 (2): 118-26.

4. Holick M. Vitamin D deficiency. N Engl J Med 2007; 357: 266-81.

5. Dusso AS, Gonzalez EA, Martin KJ. Vitamin D in chronic kideny disease. Best Pract Res Clin Endocrinol Metab 2011; 25 (4): 647-55.

6. Rizzoli R, Boonen S, Brandi ML, et al. Vitamin D supplemen- 
tation in elderly or postmenopausal women: a 2013 update of the 2008 recommendations from the European Society for Clinical and Economic Aspects of Osteoporosis and Osteoarthritis (ESCEO). Curr Med Res Opin 2013; 29 (4): 305-13.

7. Forman P, Giovannucci E, Holmes DH, et al. Plasma 25-hydroxyvitamin D levels and risk of incident hypertension. Hypertension 2007; 49: 1063-9.

8. Wang L, Song Y, Manson JE, et al. Circulating 25-hydroxyvitamin D and risk of cardiovascular disease: a meta-analysis of prospective studies. Circ Cardiovasc Qual Outcomes 2012; 5 (6): 819-29.

9. Forouhi NG, Luan J, Cooper A, et al. Baseline serum 25-hydroxy vitamin D is predictive of future glycemic status and insulin resistance. The Medica Research Cpuncil Ely Prospective Study 1990-2000. Diabetes 2008; 57: 2619-25.

10. Ginde AA, Mansbach JM, Camargo CA. Association between serum 25-hydroxyvitamin D level and upper respiratory tract infection in the Third National Health and Nutrition Examination Survey. Arch Intern Med 2009; 169 (4): 384-90.

11. Pilz S, Keinreich K, Tomaschitz A, et al. Vitamin d and cancer mortality: systematic review of prospective epidemiological studies. Anticancer Agents Med Chem 2013; 13 (1): 107-17.

12. Schottker B, Haug U, Schomburg L, et al. Strong associations of 25-hydroxyvitamin D concentrations with all-cause, cardiovascular, cancer and respiartory disease mortality in a large cohort study. Am J Clin Nutr 2013; 97 (4): 782-93.

13. Ravani P, Malberti F, Tripepi G, et al. Vitamin D levels and patient outcome in chronic kidney disease. Kidney Int 2009; 75: $88-95$.

14. Urena-Torres P, Metzger M, Haymann JP, et al. Association of kidney function, vitamin D deficiency, and circulating markers of mineral and bone disorders in CKD. Am J Kidney Dis 2011; 58 (4): 544-53.

15. Dusso AS, Gonzalez EA, Martin KJ. Vitamin D in chronic kideny disease. Best Pract Res Clin Endocrinol Metab 2011; 25 (4): 647-55.

16. Singer RF. Vitamin D in dialysis: defining deficiency and rationale of supplementation. Semin Dial 2013; 26 (1): 40-6.

17. Nigwekar SU, Bhan I, Thadhani R. Ergocalciferol and cholecalciferol in CKD. Am J Kidney Dis 2012; 60 (1): 139-56.

18. Malham M, Jorgensen SP, Lauridsen AL, et al. The effect of a single oral megadose of vitamin D provided as either ergocalciferol (D2) or cholecalciferol (D3) in alcoholic liver cirrhosis. Eur J Gastroenterol Hepatol 2012; 24 (2): 172-8.

19. Kandula P, Dobre M, Schold J, et al. Vitamin D supplementation in chronic kidney disease: a systematic review and metaanalysis of observational studies and randomized controlled trials. Clin J Am Soc Nephrol 2011; 6: 50-62.

20. Palmer SC, McGregor DO, Craig JC, et al. Vitamin D compound for people with chronic kidney disease requiring dialysis. Cochrane Database Syst Rev 2009; (4): CD005633.

21. Shroff R, Wan M, Gullett A, et al. Ergocalciferol supplementation in children with CKD delays the onset of secondary hyperparathyroidism: a randomized trial. Clin J Am Soc Nephrol 2012; 7 (2): 216-23.

22. Bucharles S, Barberato SH, Stinghen AE, et al. Impact of cholecalciferol treatment on biomarkers of inflammationand myocardial structure in hemodialysis patients without hyperparathyroidism. J Renal Nutr 2012; 22 (2): 284-91.
23. Matias PJ, Jorge C, Ferreira C, et al. Cholecalciferol supplementation in hemodialysis patients: effects on mineral metabolism, inflammation, and cardiac dimension parameters. Clin J Am Soc Nephrol 2010; 5: 905-11.

24. Pilz S, Iodice S, Zittermann A, et al. Vitamin D status and mortality risk in CKD: a meta-analysis of prospective studies. Am J Kidney Dis 2011; 58 (3): 374-82.

25. Wolf M, Shah A, Gutierrez O, et al. Vitamin D levels and early mortality among incident hemodialysis patients. Kidney Int 2007; 72: 1004-13.

26. Sprague SM, Llach F, Amdahl M, et al. Paricalcitol versus calcitriol in the treatment of secondary hyperparathyroidism. Kidney Int 2003; 63: 1483-90.

27. Coyne D, Acharya M, Qiu P, et al. Paricalcitol capsule for the treatment of secondary hyperparathyroidism in stage 3 and 4 CKD. Am J Kidney Dis 2005; 47: 263-76.

28. Becker LE, Koleganova N, Piecha G, et al. Effect of paricalcitol and calcitriol on aortic wall remodeling in uninephrectomized ApoE knockout mice. Am J Physiol Renal Physiol 2011; 300: F772-82.

29. Balint E, Marshall CF, Sprague SM. Effect of Vitamin D analogs paricalcitol and calcitriol on bone mineral in vitro. Am J Kidney Dis 2000; 36 (4): 789-96.

30. Hansen, D, Rasmussen K, Danielsen H, et al. No difference between alfacalcidol and paricalcitol in the treatment of secondary hyperparathyroidism in hemodialysis patients: a randomized crossover trial. Kidney Int 2011; 80: 841-50.

31. Joist HE, Ahya SN, Glies K, et al. Differential effects of very high doses of doxercalciferol and paricalcitol on serum phosphorus in hemodialysis patients. Clin Nephrol 2006; 65 (5): 335-41.

32. Fadem SZ, Al-Saghir F, Zoliner G, et al. Converting hemodialysis patients from intravenous paricalcitol to intravenous doxercalciferol - a dose equivalency and titration study. Clin Nephrol 2008; 70 (4): 319-24.

33. Li YC, Kong J, Wei M, et al. 1,25-Dihydroxyvitamin D3 is a negative endocrine regulator of the renin-angiotensin system. $\mathrm{J}$ Clin Invest 2002; 110: 229-38.

34. Tan X, Wen X, Liu Y. Paricalcitol inhibits renal inflammation by promoting vitamin $\mathrm{D}$ receptor-mediated sequestrtion of $\mathrm{NFkB}$ signaling. J Am Soc Nephrol 2008; 19: 1741-52.

35. de Zeeuw D, Agarwal R, Amdahl M, et al. Selective vitamin D receptor activation with paricalcitol for reduction of albuminuria in patients with type 2 diabetes (VITAL study): a randomised controlled trial. Lancet 2010; 376: 1543-51.

36. Tamez H, Zoccali C, Packhman D, et al. Vitamin D reduces left atrial volume in patients with left ventricular hypertrophy and chronic kidney disease. Am Heart J 2012; 164: 902-9.

37. Kalantar-Zadeh K, Kuwae N, Regidor DL, et al. Survival predictability of time-varying indicators of boone disease in maintenance hemodialysis patients. Kidney Int 2006; 70: 771-80.

38. Tentori F, Hunt WC, Stidley CA, et al. Mortality risk among hemodialysis patients receiving different vitamin D analogs. Kidney Int 2006; 70: 1858-65.

39. Tentori F, Albert JM, Young EW, et al. The survival advantage for haemodialysis patients taking vitamin D is questioned: findings from the Dialysis Outcomes and Practice Patterns Study. Nephrol Dial Transplant 2009; 24: 963-72.

40. Cozzolino M, Brancaccio D, Cannella G, et al. VDRA therapy 
is associated with improved survival in dialysis patients with serum intact PTH $<150 \mathrm{pg} / \mathrm{ml}$ : results of the Italian Faro survey. Nephrol Dial Transplant 2012; 27: 3588-94.

41. Vondracek SF, Hoody DW. Combination vitamin D therapy in stage 5 chornic kidney disease. Ann Pharmacother 2011; 45 (78): 1011-5.

42. Jean G, Terrat JC, Vanel T, et al. Daily oral 25-hydroxycholecalciferol supplementation for vitamin D deficiency in haemodialysis patients: effects on mineral metabolism and bone markers. Nephrol Dial Transplant 2008; 23: 3670-6.

43. Tokmak F, Quack I, Schieren G, et al. High-dose cholecalciferol to correct vitmain D deficiency in haemodialysis patients. Nephrol Dial Transplant 2008; 23: 3016-20.

44. Armas LA, Andukuri R, Barger-Lux J, et al. 25-hydroxyvitamin $D$ response to cholecalciferol supplementation in hemodialysis. Clin J Am Soc Nephrol 2012; 7: 1428-34.

45. Matias PJ, Jorge C, Ferreira C, et al. Cholecalciferol supplementation in hemodialysis patients: effects on mineral metabolism, inflammation, and cardiac dimension parameters. Clin J Am Soc Nephrol 2010; 5: 905-11.

46. Wasse H, Huang R, Long Qi, et al. Efficacy and safety of a short course of very-high-dose cholecalciferol in hemodialysis. Am J Clin Nutr 2012; 95: 522-8.

47. Delanaye P, Weekers L, Warling X, et al. Cholecalciferol in haemodialysis patients: a randomized, double-blind, proof-ofconcept and safety study. Nephrol Dial Transplant 2013.

48. Wesseling-Perry K, Pereira RC, Sahney S, et al. Calcitriol and doxercalciferol are equivalent in controlling bone turnover, suppressing parathyroid hormone, and increasing fibroblast growth factor-23 in secondary hyperparathyroidism. Kidney Int 2011; 79: 112-9.

49. Hansen D, Rasmussen K, Pedersen S, et al. Changes in fibroblast growth factor 23 during treatment of secondary hyperparathyroidism with alfacalcidol or paricalcitol. Nephrol Dial Transplant 2012; 27 (6): 2263-9. 